\title{
Review
}

Korean J Obes 2016 September;25(3):115-120

http://dx.doi.org/10.7570/kjo.2016.25.3.115

pISSN 2383-899X elSSN 2234-7631

\section{Measurements of Adiposity and Body Composition}

\author{
Chong Hwa Kim* \\ Department of Internal Medicine, Sejong General Hospital, Bucheon, Korea
}

Obesity is defined as an excess accumulation of body fat. Measuring body fat accurately is difficult, and no method is easily available for routine clinical use. Common methods of exploring the levels of adiposity include body mass index (BMI), waist circumference, skinfolds, bioelectrical impedance analysis, dual energy x-ray absorptiometry (DEXA), computerized tomography (CT), and magnetic resonance imaging (MRI). Traditionally, overweight or obese status has been evaluated by anthropometric measurement of weight-for-height. More recently, BMI has been used. The normal range is $19-24.9 \mathrm{~kg} / \mathrm{m}^{2}$, overweight is $25-29.9 \mathrm{~kg} / \mathrm{m}^{2}$, and obese is $\geq 30 \mathrm{~kg} / \mathrm{m}^{2}$. However, the appropriateness of this definition among Asian individuals has been questioned. Asian individuals generally have a higher percentage of body fat than do Western individuals at the same BMI levels. Consequently, for the Korean population, overweight status is defined based on an increase in morbidity and obesity based on an increase in mortality; overweight is defined as a BMI of $23-24.9 \mathrm{~kg} / \mathrm{m}^{2}$ and obese as a BMI $\geq 25 \mathrm{~kg} / \mathrm{m}^{2}$. Simple anthropometric measurements can be used, such as waist circumference. A waist circumference greater than $102 \mathrm{~cm}$ in men and $88 \mathrm{~cm}$ in women is a risk factor for insulin resistance, diabetes mellitus and cardiovascular disease. Based on the World Health Organization (WHO) recommendations, the waist circumference (WC) cutoff for Asians was suggested as $90 \mathrm{~cm}$ for men and $80 \mathrm{~cm}$ for women, respectively, which was different from that of other ethnic groups, such as Europeans. For Koreans, abdominal obesity is defined as a WC $\geq 90 \mathrm{~cm}$ for men and $\geq 85 \mathrm{~cm}$ for women.

Key words: Adiposity, Body composition, Measurement

\section{Introduction}

Obesity is often defined as a condition of abnormal or excessive fat accumulation in adipose tissue, to the extent that health may be impaired. ${ }^{1}$ Obesity is associated with increased risks of type 2 diabetes mellitus (T2DM), hypertension, dyslipidemia, metabolic syndrome, coronary heart disease, other atherosclerotic diseases, non-alcoholic steatohepatitis, and gout. ${ }^{2}$ The underlying disease is the undesirable positive energy balance and weight gain. However, obese individuals differ not only in the amount of excess fat that they store, but also in the regional distribution of that fat within the body. The distribution of fat induced by weight gain affects the risks associated with obesity, and the types of disease that result. Indeed, excess abdominal fat is generally as great a risk factor for disease as is excess body fat. It is useful, therefore, to be able to distinguish between those at increased risk as a result of "abdominal fat distribution", or "android obesity" as it is often known, from those with the less serious "gynoid" fat distribution, in which fat is more evenly and peripherally distributed around the body. What is the best way to determine whether a body is fat or fit? Body fat can be measured in several ways, with each body fat assessment method having pros and cons. Common methods of exploring the levels of adiposity include body mass index (BMI), waist circumference, skinfolds, bioelectrical impedance analysis, dual energy x-ray absorptiometry (DEXA), computerized tomography (CT) and magnetic resonance imaging (MRI). ${ }^{3}$ Below is a brief overview of some of the most popular methods for measuring body fat, from basic body measurements to high-tech body scans, along with their strengths and limitations. In addition, we outline the most appropriate methods for: (1) classifying overweight and obese status in adults; and (2) identifying abdominal fat distribution.

\section{Body mass index $(\mathrm{BMI})^{3}$}

BMI is the ratio of weight to height, calculated as weight $(\mathrm{kg}) /$
Corresponding author Chong Hwa Kim (iD http://orcid.org/0000-0002-4563-7772 Department of Internal Medicine, Sejong General Hospital, 28 Hohyeon-ro 489beon-gil, Sosa-gu, Bucheon 14754, Korea

Tel: +82-32-340-1116 Fax: +82-32-340-1005 E-mail: drangelkr@hanmail.net
Copyright () 2016 Korean Society for the Study of Obesity

(c) This is an Open Access article distributed under the terms of the Creative Commons Attribution Non-Commercial License (http://creativecommons.org/licenses/by-nc/4.0/) which permits unrestricted non-commercial use, distribution, and reproduction in any medium, provided the original work is properly cited. 
height $\left(\mathrm{m}^{2}\right)$, or weight (lb)/height $\left(\mathrm{in}^{2}\right)$ multiplied by 703 .

Strengths: BMI is easy to measure, inexpensive, and provides standardized cutoff points for overweight and obese status. Normal weight is a BMI between 18.5 and 24.9; overweight is a BMI between 25.0 and 29.9; obese is a BMI of 30.0 or higher. BMI is strongly correlated with body fat levels, as measured by the most accurate methods; hundreds of studies show that a high BMI predicts higher risk of chronic disease and early death.

Limitations: BMI involves indirect and imperfect measurement, and does not distinguish between body fat and lean body mass. It is not as accurate a predictor of body fat in the elderly as it is in younger and middle-aged adults. At the same BMI, women have, on average, more body fat than men, and Asians have more body fat than whites.

\section{Waist circumference ${ }^{3}$}

Waist circumference is the simplest and most common way to measure abdominal obesity. The extra fat found around the middle is an important factor in health, even independent of BMI. It is the circumference of the abdomen, measured at the natural waist (in between the lowest rib and the top of the hip bone), the umbilicus (belly button), or at the narrowest point of the midsection.

Strengths: It is easy to measure, inexpensive, and is strongly correlated with body fat in adults as measured by the most accurate methods. Studies show that waist circumference predicts the development of disease and death.

Limitations: The measurement procedure has not been standardized, and there is a dearth of good comparison standards (reference data) for waist circumference in children. It may be difficult to measure and less accurate in individuals with a BMI of 35 or higher.

\section{Waist-to-hip ratio ${ }^{3}$}

Like the waist circumference, the waist-to-hip ratio (WHR) is also used to measure abdominal obesity. It is calculated by measuring the waist and the hip (at the widest diameter of the buttocks), and then dividing the waist measurement by the hip measurement.

Strengths: WHR has a good correlation with body fat as measured by the most accurate methods, and is inexpensive. Studies show that waist-to-hip ratio predicts the development of disease and death in adults.
Limitations: WHR is more prone to measurement error because it requires two measurements, and it is more difficult to measure hips than it is to measure waists. In addition, it is more complex to interpret than waist circumference, since increased waist-to-hip ratio can be caused by increased abdominal fat or decreased lean muscle mass around the hips. Turning the measurements into a ratio leads to a loss of information; two people with very different BMIs could have the same WHR. It may be difficult to measure and less accurate in individuals with a BMI of 35 or higher.

\section{Skinfold thickness ${ }^{3}$}

In this method, researchers use a special caliper to measure the thickness of a "pinch" of skin and the fat beneath it in specific areas of the body (the trunk, the thighs, front and back of the upper arm, and under the shoulder blade). Equations are used to predict body fat percentage based on these measurements.

Strengths: It is convenient, safe, inexpensive, portable, fast and easy (except in individuals with a BMI of 35 or higher).

Limitations: It is not as accurate or reproducible as other methods, and very difficult to measure in individuals with a BMI of 35 or higher.

\section{Bioelectric impedance (BIA) ${ }^{3}$}

BIA equipment sends a small, imperceptible, safe electric current through the body, measuring the resistance. The current faces more resistance passing through body fat than it does passing through lean body mass and water. Equations are used to estimate body fat percentage and fat-free mass.

Strengths: BIA is convenient, safe, relatively inexpensive, portable, fast and easy.

Limitations: It is difficult to calibrate. The ratio of body water to fat may change during illness, dehydration or weight loss, decreasing accuracy. It is not as accurate as other methods, especially in individuals with a BMI of 35 or higher.

\section{Underwater weighing (Densitometry) ${ }^{3}$}

Individuals are weighed in air and while submerged in a tank. Researchers use formulas to estimate body volume, body density, and 
body fat percentage. Fat is more buoyant (less dense) than water, so an individual with high body fat will have a lower body density than an individual with low body fat. This method is typically only used in a research setting.

Strengths: It is very accurate.

Limitations: It is time consuming, and requires individuals to be submerged in water. It is generally not a good option for children, older adults, and individuals with a BMI of 40 or higher.

\section{Air-displacement plethysmography ${ }^{3}$}

This method uses a similar principle to underwater weighing but can be done in the air instead of in water. Individuals sit in a small chamber wearing a bathing suit; one commercial example is the "Bod Pod." The machine estimates body volume based on air pressure differences between the empty chamber and the occupied chamber.

Strengths: It is relatively quick and comfortable, accurate, safe, and a good choice for children, older adults, pregnant women, individuals with a BMI of 40 or higher, and other individuals who would not want to be submerged in water.

Limitations: It is expensive.

\section{Dilution method (Hydrometry) $)^{3}$}

Individuals drink isotope-labeled water and give body fluid samples. Researchers analyze these samples for isotope levels, which are then used to calculate total body water, fat-free body mass, and in turn, body fat mass.

Strengths: It is relatively low cost, accurate, and safe. It can be used in individuals with a BMI of 40 or higher, as well as in children and pregnant women.

Limitations: The ratio of body water to fat-free mass may change during illness, dehydration, or weight loss, decreasing accuracy.

\section{Dual energy $\mathrm{x}$-ray absorptiometry ${ }^{3}$}

X-ray beams pass through different body tissues at different rates. Therefore, DEXA uses two low-level X-ray beams to develop estimates of fat-free mass, fat mass, and bone mineral density. DEXA is typically only used for this purpose in research settings.

Strengths: DEXA is accurate.

Limitations: Equipment is expensive and cannot be moved, and DEXA cannot accurately distinguish between different types of fat (fat under the skin, also known as "subcutaneous" fat vs. fat around the internal organs, or "visceral" fat). It cannot be used with pregnant women, since it requires exposure to a small dose of radiation. Most current systems cannot accommodate individuals with a BMI of 35 or higher.

\section{Computerized tomography and magnetic resonance imaging ${ }^{3}$}

These two imaging techniques are now considered to be the most accurate methods for measuring tissue, organ, and whole-body fat mass, as well as lean muscle mass and bone mass. CT and MRI scans are typically only used for this purpose in research settings.

Strengths: They are accurate and allow for measurement of specific body fat compartments, such as abdominal fat and subcutaneous fat.

Limitations: Equipment is extremely expensive and cannot be moved. CT scans cannot be used with pregnant women or children, due to the high amounts of ionizing radiation used. Some MRI and CT scanners may not be able to accommodate individuals with a BMI of 35 or higher.

\section{Why classify overweight and obese status?}

The graded classification of overweight and obese status is valuable for a number of reasons. In particular, it allows for meaningful comparisons of weight status within and between populations, the identification of individuals and groups at increased risk of morbidity and mortality, the identification of priorities for intervention at individual and community levels, and a firm basis for evaluating interventions.

BMI is a simple index of weight-for-height that is commonly used to classify underweight, overweight and obese status in adults. It is defined as the weight in kilograms divided by the square of the height in meters $\left(\mathrm{kg} / \mathrm{m}^{2}\right)$.

The classification of overweight and obese, according to BMI, is shown in Table 1 . Obese is classified as a BMI $\geq 30.0$. The classification shown in Table 1 is in agreement with that recommended by the 
Table 1. Classification of adults according to $\mathrm{BMI}^{*}$

\begin{tabular}{lll}
\hline Classification & \multicolumn{1}{c}{ BMl } & \multicolumn{1}{c}{ Risk of comorbidities } \\
\hline Underweight & $<18.5$ & Low (but risk of other clinical problems increased) \\
Normal range & $18.50-24.99$ & Average \\
Asia-normal range & $18.5-22.9$ & Average \\
Overweight & $\geq 25.00$ & \\
Pre-obese & $25.00-29.99$ & Increased \\
Asia-overweight & $23-24.9$ & \\
Obese class I & $30.00-34.99$ & Moderate \\
Asia-obese class I & $25-29.9$ & \\
Obese class II & $35.00-39.99$ & Severe \\
Asia-class II & $\geq 30.00$ & \\
Obese class III & $\geq 40.00$ & Very severe \\
\hline
\end{tabular}

*These BMl values are age-independent and the same for both sexes. However, BMI may not correspond to the same degree of fatness in different populations due, in part, to differences in body proportions. The table shows a simplistic relationship between $\mathrm{BMl}$ and the risk of comorbidity, which can be affected by a range of factors, including the nature of an individual's diet, ethnic group and activity level. The risks associated with increasing BMl are continuous and graded and begin at a BMl above 25. The interpretation of BMl gradings in relation to risk may vary for different populations. Both $\mathrm{BMl}$ and a measure of fat distribution (waist circumference or waist-hip ratio ) are important in calculating the risk of obesity-related comorbidities.

$\mathrm{WHO}^{4}$, but includes an additional subdivision at BMI 35.0-39.9 in recognition of the fact that management options for dealing with obesity differ when a BMI is above 35 . The WHO classification is based primarily on the association between BMI and mortality. However, the appropriateness of this definition in Asian populations has been questioned. Asian individuals generally have a higher percentage of body fat than do Western individuals at the same BMI levels. ${ }^{5}$ Consequently, the WHO recommends that cutoff values for the definition of overweight and obese should be lower for Asian individuals than for Western individuals. ${ }^{6}$

\section{Use of other cut-off points in the classification of obesity}

A BMI of 30 or more is now widely accepted as denoting obesity. Differences in cut-off points have a major impact on estimates of the prevalence of obesity. For meaningful comparisons between or within populations, it is therefore advisable to use the single BMI cut-off points proposed in Table 1 . The WHO recommends that cutoff values for the definition of overweight and obese should be lower for Asian populations than for White populations. ${ }^{6}$ In a prospective cohort study of more than 1 million Koreans, the average baseline BMI in both sexes was 23.2, and the death rate from any cause had a Jshaped association with the BMI, regardless of cigarette-smoking history. ${ }^{7}$ The risk of death from any cause was lowest among patients with a BMI of 23.0 to 24.9. In cohorts of East Asians, including Chinese, Japanese, and Korean individuals, the lowest risk of death was seen among persons with a BMI of 22.6 to $27.5{ }^{8}$ There is a significantly increased risk of T2DM and hypertension among those with a BMI of 23 to 24.9, as compared to those with a BMI within the normal range. These BMI cutoffs are arbitrary, along a continuum of increasing risk with increasing BMI. The cutoffs used for the definition of obesity are based on the excess morbidity and mortality associated with increasing body fat content. ${ }^{6}$ Consequently, for Korean individuals, we defined overweight status based on an increase in morbidity and obese status based on an increase in mortality: overweight is defined as a BMI of 23 to $24.9 \mathrm{~kg} / \mathrm{m}^{2}$ and obese as a BMI $\geq 25 \mathrm{~kg} / \mathrm{m}^{2}$.

\section{Use of BMI to classify obesity}

BMI can be considered to provide the most useful, albeit crude, population-level measure of obesity. The robust nature of the measurements and the widespread routine inclusion of weight and height in clinical and population health surveys suggest that a more selective measure of adiposity, such as skinfold thickness measurements, provides additional rather than primary information. BMI can be used to estimate the prevalence of obesity within a population and the risks associated with it; it does not, however, account for the wide variation in the nature of obesity between different individuals and populations.

\section{Waist circumference and waist: hip ratio}

Abdominal fat mass can vary dramatically within a narrow range of total body fat or BMI. Indeed, for any accumulation of total body fat, men have on average twice the amount of abdominal fat than is generally found among premenopausal women. ${ }^{9}$ Other methods, in addition to the measurement of BMI, would therefore be valuable in identifying individuals at increased risk for obesity-related illnesses due to abdominal fat accumulation.

The most commonly used cutoffs among Caucasians for waist circumference (WC) are $102 \mathrm{~cm}$ for men and $88 \mathrm{~cm}$ for women. Over the past 10 years, it has become accepted that a high WHR (WHR $>1.0$ in men and $>0.85$ in women) indicates abdominal fat accumulation. ${ }^{10}$ However, recent evidence suggests that waist circumference 
alone - measured at the midpoint between the lower border of the rib cage and the iliac crest - may provide a more practical correlate of abdominal fat distribution and associated poor health. ${ }^{11-13}$

Waist circumference is a convenient and simple measurement that is unrelated to height10, correlates closely with BMI and $\mathrm{WHR}^{11}$ and is an approximate index of intra-abdominal fat mass ${ }^{12-14}$ and total body fat. ${ }^{15}$ Furthermore, changes in waist circumference reflect changes in risk factors for cardiovascular disease (CVD) ${ }^{16}$ and other forms of chronic disease, even though these risks appear to vary in different populations.

Based on the WHO recommendations, the WC cutoff for Asians was suggested as $90 \mathrm{~cm}$ for men and $80 \mathrm{~cm}$ for women, which was different from that of other ethnic groups, such as Europeans. 6 However, when the WHO Asia-Pacific criteria were applied, the prevalence of abdominal obesity in Korea was $20 \%$ in men and $40 \%$ in women, indicating the prevalence of abdominal obesity in women was two times higher than in men. Therefore, a study of the WC cutoff points for defining abdominal obesity in Korean individuals was performed using data from the Korean National Health and Nutritional Examination Survey 1998. The optimal WC to predict the risk factors of metabolic syndrome in Koreans was 82 to $84 \mathrm{~cm}$ for men, and 79 to $82 \mathrm{~cm}$ for women. ${ }^{17} \mathrm{WC}$ in the 80 th percentile in the Korean population is 90 and $86.5 \mathrm{~cm}$ for men and women, respectively. In Korea, abdominal obesity is defined as a WC $\geq 90 \mathrm{~cm}$ for men and $\geq 85 \mathrm{~cm}$ for women.

\section{Conclusion}

Obesity can be defined as a disease in which excess body fat has accumulated to such an extent that health may be adversely affected. However, the amount of excess fat, its distribution within the body, and the associated health consequences vary considerably among obese individuals. What is the best way to determine whether a body is fat or fit? Body fat can be measured in several ways, with each body fat assessment method having pros and cons. Common methods of exploring the levels of adiposity include BMI, waist circumference, skinfolds, bioelectrical impedance analysis, DEXA, CT and MRI. The graded classification of overweight and obese status permits meaningful comparisons of weight status within and between populations, and makes it possible to identify individuals and groups at an increased risk of morbidity and mortality. Obese individuals with excess fat in the intra-abdominal depots are at particular risk of the adverse health consequences of obesity. Therefore, measurement of waist circumference provides a simple and practical method of identifying overweight patients at increased risk of obesity-associated illness due to abdominal fat distribution.

\section{Conflicts of Interest}

I had no conflict of interest in this paper.

\section{References}

1. Garrow JS. Obesity and related diseases. London: Churchill Livingstone; 1988 p. 1-16.

2. Park JH, Yoon SJ, Lee H, Jo HS, Lee SI, Kim Y, et al. Burden of disease attributable to obesity and overweight in Korea. Int J Obes (Lond) 2006;30:1661-9.

3. $\mathrm{Hu}$ F. Measurements of adiposity and body composition. In: $\mathrm{Hu}$ FB, editor. Obesity epidemiology. New York: Oxford University Press; 2008. p. 53-83.

4. World Health Organization. Physical status: the use and interpretation of anthropometry, report of a WHO expert committee. Geneva: World Health Organization; 1995.

5. Swinburn BA, Craig PL, Daniel R, Dent DP, Strauss BJ. Body composition differences between Polynesians and Caucasians assessed by bioelectrical impedance. Int J Obes Relat Metab Disord 1996;20:889-94.

6. Bassett J; International Diabetes Institute; World Health Organization Regional Office for the Western Pacific; International Association for the Study of Obesity; International Obesity Task Force. The Asia-Pacific perspective: redefining obesity and its treatment. Melbourne: Health Communications Australia; 2000.

7. Jee SH, Sull JW, Park J, Lee SY, Ohrr H, Guallar E, et al. Bodymass index and mortality in Korean men and women. N Engl J Med 2006;355:779-87.

8. Zheng W, McLerran DF, Rolland B, Zhang X, Inoue M, Matsuo K, et al. Association between body-mass index and risk of death in more than 1 million Asians. N Engl J Med 2011;364:719-29.

9. Lemieux S, Prud'homme D, Bouchard C, Tremblay A, Després JP. Sex differences in the relation of visceral adipose tissue accumulation to total body fatness. Am J Clin Nutr 1993;58:463-7. 
10. Han TS, Seidell JC, Currall JE, Morrison CE, Deurenberg P, Lean ME. The influences of height and age on waist circumference as an index of adiposity in adults. Int J Obes Relat Metab Disord 1997;21:83-9.

11. James WPT. The epidemiology of obesity. In: Chadwick DJ, Cardew GC, editors. The origins and consequences of obesity. Chichester: John Wiley \& Sons; 1996. p. 116.

12. Seidell JC. Are abdominal diameters abominable indicators? In: Angel A, Bouchard C, editors. Progress in Obesity Research: 7. London: Libbey; 1995. p. 305-8.

13. Lean ME, Han TS, Morrison CE. Waist circumference as a measure for indicating need for weight management. BMJ 1995;311: $158-61$.
14. Ross R, Léger L, Morris D, de Guise J, Guardo R. Quantification of adipose tissue by MRI: relationship with anthropometric variables. J Appl Physiol (1985) 1992;72:787-95.

15. Lean ME, Han TS, Deurenberg P. Predicting body composition by densitometry from simple anthropometric measurements. Am J Clin Nutr 1996;63:4-14.

16. Han TS, Richmond P, Avenell A, Lean ME. Waist circumference reduction and cardiovascular benefits during weight loss in women. Int J Obes Relat Metab Disord 1997;21:127-34.

17. Lee S, Park HS, Kim SM, Kwon HS, Kim DY, Kim DJ, et al. Cutoff points of waist circumference for defining abdominal obesity in the Korean population. Korean J Obes 2006;15:1-9. 